\title{
Space Charge Measurements on Multi-dielectrics by Means of the Pulsed Electroacoustic Method
}

\author{
R. Bodega, P.H.F. Morshuis and J.J. Smit \\ Delft University of Technology \\ Mekelweg, 4-2628 CD Delft, The Netherlands
}

\begin{abstract}
The Pulsed Electroacoustic (PEA) method is now widely accepted as one of the most simple and effective techniques for the measurement of the dynamic space charge distribution in solid dielectrics. Recently, the PEA method has been applied also to laminar test objects composed of two or more layers of different dielectrics (multidielectrics). However, when a multi-dielectric is tested by means of the PEA method, the different acoustic and electric properties of the materials affect the detected space charge signal. In this paper, the principle of the PEA technique is reviewed in case the test object is a multi-dielectric. The generation, transmission and reflection of electrically-induced acoustic waves are described. Based on the proposed approach, results of PEA measurements performed on various kinds of multi-dielectrics are presented and discussed.
\end{abstract}

Index Terms - Space charge, interface phenomena, pulsed electroacoustic method, PEA, dielectric materials, acoustic impedance, acoustic signal detection.

\section{LIST OF SYMBOLS}

\begin{tabular}{|c|c|c|}
\hline Symbol & Variable & Unit \\
\hline$a$ & Electrostrictive coefficient & - \\
\hline$b$ & Width of a slab of space charge & $\mathrm{m}$ \\
\hline$D$ & Electric flux density & $\mathrm{C} \cdot \mathrm{m}^{-2}$ \\
\hline$d$ & Insulation thickness & $\mathrm{m}$ \\
\hline E & Electric field & $\mathrm{V} \cdot \mathrm{m}^{-1}$ \\
\hline $\bar{E}$ & Constant electric field & $\mathrm{V} \cdot \mathrm{m}^{-1}$ \\
\hline$e_{p}$ & Pulsed electric field & $\mathrm{V} \cdot \mathrm{m}^{-1}$ \\
\hline$f$ & Force per unit volume & $\mathrm{N} \cdot \mathrm{m}^{-3}$ \\
\hline$G$ & Generation coefficient of acoustic waves & - \\
\hline$p$ & Electrostatic pressure & $\mathrm{Pa}$ \\
\hline$p^{\sim}$ & Transient part of the electrostatic pressure & $\mathrm{Pa}$ \\
\hline$p^{\sim}$ & $\begin{array}{l}\text { Transmitted component of the transient part of the } \\
\text { electrostatic pressure }\end{array}$ & $\mathrm{Pa}$ \\
\hline$p^{\sim \prime \prime}$ & $\begin{array}{l}\text { Reflected component of the transient part of the } \\
\text { electrostatic pressure }\end{array}$ & $\mathrm{Pa}$ \\
\hline$p_{T O T}$ & Total pressure distribution & $\mathrm{Pa}$ \\
\hline$R$ & Reflection coefficient of acoustic waves & - \\
\hline$T$ & Transmission coefficient of acoustic waves & - \\
\hline$t$ & Time & $\mathrm{s}$ \\
\hline$U_{0}$ & Dc external voltage & $\mathrm{V}$ \\
\hline$u_{p}$ & Pulsed voltage & $\mathrm{V}$ \\
\hline$v$ & Speed of sound & $\mathrm{m} \cdot \mathrm{s}^{-1}$ \\
\hline$x$ & Distance & $\mathrm{m}$ \\
\hline$Z$ & Acoustic impedance & $\mathrm{kg} \cdot \mathrm{m}^{-2} \cdot \mathrm{s}^{-1}$ \\
\hline$\delta$ & Density & $\mathrm{kg} \cdot \mathrm{m}^{-3}$ \\
\hline$\varepsilon_{0}$ & Vacuum permittivity & $\mathrm{F} \cdot \mathrm{m}^{-1}$ \\
\hline$\varepsilon_{r}$ & Relative permittivity & - \\
\hline$\kappa$ & Interfacial charge density & $\mathrm{C} \cdot \mathrm{m}^{-2}$ \\
\hline$\rho$ & Space charge density & $\mathrm{C} \cdot \mathrm{m}^{-3}$ \\
\hline$\Pi$ & Permanent dipole density & $\mathrm{C} \cdot \mathrm{m}^{-2}$ \\
\hline$\tau$ & Delay time & $\mathrm{s}$ \\
\hline
\end{tabular}

Manuscript received on 11 May 2005, in final form 7 September 2005.

\section{INTRODUCTION}

IN high voltage insulation systems, combinations of insulating materials are often used. As a consequence, dielectric interfaces are encountered at the boundary between adjacent dielectrics. A typical example of a combination of dielectrics used in practice is found in cable accessories (i.e. joints and terminations), where a solid-solid interface is present between the insulation of the cable and that of the accessory. Especially under dc voltage stressing conditions, dielectric interfaces are a favorite location for charge build-up. In fact, according to the Maxwell-Wagner theory for interfacial polarization [1], charge accumulates at interfaces if the ratio of the conductivities and that of the permittivities of the two media in contact are not the same. In addition, because of the characteristic properties of the interface (e.g. smoothness of the surfaces, contact pressure, presence of lubricants or chemical treatments and differences between bulk and surface conduction processes), a more complex build-up of interfacial charge may occur [2-7]. The presence of space charge within an insulating material distorts the original Laplacian field. This causes a local field enhancement in the material that may lead to accelerated insulation degradation and, ultimately, to electrical breakdown. For this reason, space charge measurements represent a significant tool for dielectric interface characterization [4], especially at dc voltages.

In order to investigate the space charge behavior of dielectric interfaces, experimental studies have been carried out recently on multi-dielectric specimens. In most of the 
cases, the PEA method $[8,9]$ was used for this goal. Hitherto, several kinds of multi-dielectrics have been tested by means of the PEA method [2-7, 10-14], but only little attention has been paid to the fact that, generally, the PEA method does not directly provide the space charge distribution within the sample if the sample is composed of different insulation layers [15-20].

There are two main reasons why the output signal of a PEA measurement does not correspond to the space charge distribution within a multi-dielectric. Firstly, a pulsed voltage is applied to the insulation to be tested. This pulse induces a transient force distribution within the multi-dielectric which originates the acoustic signal to be detected. Assuming that the layers of the multi-dielectric have different electrical properties, the transient force distribution not only depends on the space charge, but also on the permittivity of the layers. Secondly, the PEA method is based on generation and propagation of acoustic waves. In the case of different acoustic properties of the materials in contact (acoustical mismatching), waves experience different generation, transmission and reflection coefficients. Then, reflections can occur at the interfaces between two different materials, leading to possible misjudgment of measurement results. In order to correctly evaluate the space charge distribution in a multidielectric by means of PEA measurements, the relation between both location and magnitude of space charge and detected signal must be known.

To contribute to this discussion, the authors studied the electro-acoustic phenomena occurring in a multi-dielectric tested by means of the PEA method. A procedure for the interpretation of results is given. Based on this procedure, experimental results are interpreted and discussed.

\section{ELECTRICALLY-INDUCED PRESSURE DISTRIBUTION}

In the PEA method $[8,9]$, a pulsed voltage $u_{p}(\mathrm{t})$, which is superimposed on the poling dc voltage $U_{0}$, is applied across a test object (e.g. a flat specimen). Depending on the sample thickness, typical values for the amplitude and width of the pulse are $0.1-4 \mathrm{kV}$ and 5-200 ns, respectively. The application of the pulse leads to a modification of the electrostatic force distribution acting on the sample and as a result stress (acoustic) waves are generated. Those waves are detected by a sensor after having traveled through the sample and through the earth electrode. In Figure 1, the PEA principle is schematically represented. In the following, the magnitude of the generated pressure waves is calculated for a situation in which two flat insulating materials, A and B, are placed in between electrode-1, which is connected to high voltage, and electrode-2, which is connected to earth, see Figure 2.

A general expression for the force $f$ for a unit volume, which acts on a dielectric when it is stressed with an electric field $E$, may be derived thermodynamically, e.g. [21]:

$$
f=\rho \cdot E-\frac{\varepsilon_{0}}{2} \cdot E^{2} \cdot \nabla \varepsilon_{r}-\frac{\varepsilon_{0}}{2} \cdot \nabla\left(E^{2} \cdot a\right)+\Pi \cdot \nabla E
$$

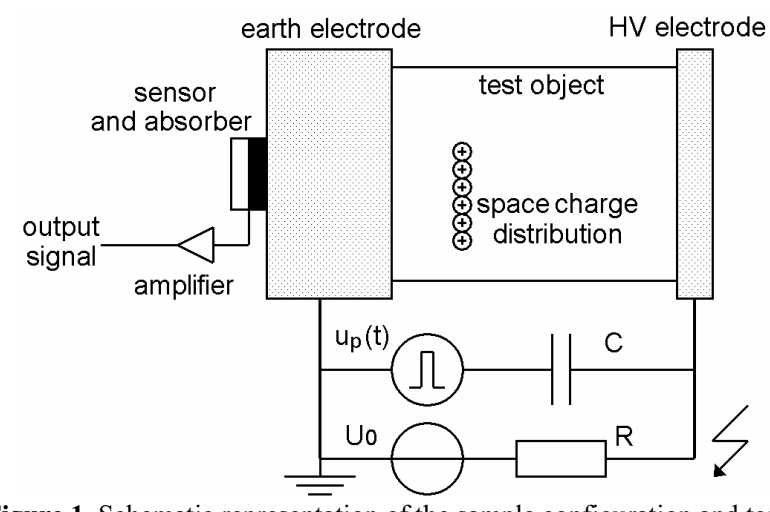

Figure 1. Schematic representation of the sample configuration and test set-up for space charge measurements by means of the PEA method.

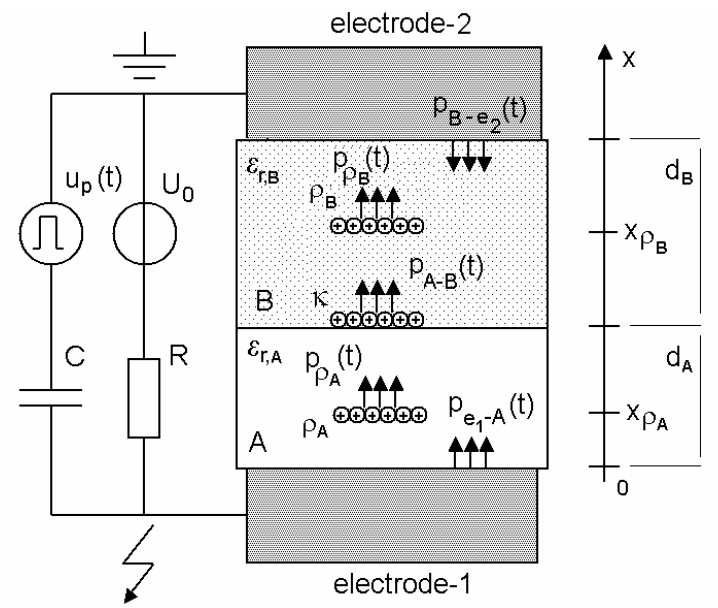

Figure 2. Electrically-induced pressure distribution in a multi-dielectric tested by means of the PEA method.

In (1), $\rho$ is the distribution of free space charges, $\varepsilon_{0}$ the vacuum permittivity, $\varepsilon_{r}$ the relative permittivity of the dielectric, $a$ the electrostrictive coefficient and $\Pi$ the permanent dipole density. The force for a unit volume is the sum of four contributions. The first term represents the force acting on a distribution of free space charges $\rho$ embedded in the dielectric. Analogously, a force per unit area acts on a distribution of surface charges $\kappa$ present within the material. The second term of (1) will contribute to the force where the dielectric is not homogeneous. As a consequence, a force per unit volume is originated at the interface between two different dielectrics if an external field is applied. Because of this fact, the PEA method generally provides a signal at the dielectric interface of a multi-dielectric, where a discontinuity of the permittivity exists. This phenomenon occurs even in the situation in which neither space charge nor interfacial charge are present within the multi-dielectric. The third term represents the so called electrostriction term. This takes into account the volume force the dielectric experiences in a nonuniform electric field, due to the variation of the relative permittivity with strain, represented in (1) by the electrostrictive coefficient $a$. The last term must be taken into account only if permanent dipoles are present in the dielectric (e.g. in case of piezoelectric materials). So, in general, electric forces due to space charges are not the only sources of the signal provided by elastic methods for space charge 
measurements [22-24]. However, considering that the materials $\mathrm{A}$ and $\mathrm{B}$ of the multi-dielectric in Figure 2 do not show any permanent dipoles, and assuming that the poling field is uniform because of the particular electrode-sample geometry, only the first two terms of (1) contribute to the force density in the studied configuration.

By integration of (1) along the $x$-direction, as defined in Figure 2, the electrostatic pressure $p(x, t)$ is given. The result is a spatial distribution of the electrostatic pressure which is different from zero only at specific locations of the multidielectric:

- $p_{e_{1}-A}(t)$ at the interface between electrode-1 and material-A;

- $p_{B-e 2}(t)$ at the interface between material-B and electrode-2;

- $p_{A-B}(t)$ at the interface between material-A and material-B (because of the discontinuity of the permittivity and the possible presence of interfacial charge $\kappa)$;

- $p_{\rho_{A}}(t)$ (or $p_{\rho_{B}}(t)$ ) at the space charge $\rho_{A}$ (or $\rho_{B}$ ) location.

The acoustic signal detected by the sensor is the transient component $\widetilde{p}(x, t)$ of the total electrostatic pressure $p(x, t)$. The transient component of the electrostatic pressure will be calculated in the next subsections, with the following assumptions:

- the pulsed field in both A and B layers can be expressed as:

$$
\begin{aligned}
& e_{p, A}(t)=\frac{\varepsilon_{r, B}}{\varepsilon_{r, A} \cdot d_{B}+\varepsilon_{r, B} \cdot d_{A}} \cdot u_{p}(t) \\
& e_{p, B}(t)=\frac{\varepsilon_{r, A}}{\varepsilon_{r, A} \cdot d_{B}+\varepsilon_{r, B} \cdot d_{A}} \cdot u_{p}(t)
\end{aligned}
$$

where $d_{A}$ and $d_{B}$ are the thickness of layers $\mathrm{A}$ and $\mathrm{B}$ whereas $\varepsilon_{r, A}$ and $\varepsilon_{r, B}$ are the relative permittivities of $\mathrm{A}$ and $\mathrm{B}$;

- the electric field $\bar{E}$, which is due to the applied voltage, the space charge and the interfacial charge, is assumed to be constant and is derived from equations (4) and (5):

$U_{0}=-\nabla \bar{E}$

$\rho=\nabla \cdot\left(\varepsilon_{0} \varepsilon_{r} \bar{E}\right)$

- the constant field $\bar{E}$ is much bigger than the pulsed field;

- the mobility of the space charge $\rho$ is sufficiently low to make this charge detectable by means of the PEA method.

In the following sections, the transient pressure distributions are deduced for a number of simple cases. More complicated cases can then be handled as a superimposition of several simple cases.

\subsection{VOLTAGE APPLIED IN ABSENCE OF SPACE CHARGE AND INTERFACIAL CHARGE}

Figure 3 represents the pressure distribution when a dc voltage is applied to the multi-dielectric in the absence of space charge and interfacial charge. In practice, this situation occurs during the first instants after the application of the dc voltage, when the electric field can be considered capacitively distributed. After a certain time, depending on the insulation properties and on the test conditions, charges accumulate within the insulation, leading to a resistive distribution of the field.

The electric field $E_{0, A}$ in the A-layer due to the dc voltage $U_{0}$ can be written as:

$$
\bar{E}_{0, A}=\frac{\varepsilon_{r, B}}{\varepsilon_{r, A} \cdot d_{B}+\varepsilon_{r, B} \cdot d_{A}} \cdot U_{0}
$$

A similar expression can be found for the field $\bar{E}_{0, B}$ in the Blayer. By considering the total field acting on the multidielectric as the sum of the dc field $E_{0}$ and the pulsed field $e_{p}(t)$, the pressure at the electrode- $1 /$ material-A interface becomes:

$p_{0, e_{1}-A}(t)=\frac{1}{2} \cdot \varepsilon_{0} \cdot \varepsilon_{r, A} \cdot\left(\bar{E}_{0, A}+e_{p, A}(t)\right)^{2}$

Combining (2), (6) and (7) and considering $E_{0}>>e_{p}$, the transient component $\widetilde{p}_{0, e_{1}-A}(t)$ of the pressure $p_{0, e_{1}-A}(t)$ becomes:

$\widetilde{p}_{0, e_{1}-A}(t)=\frac{\varepsilon_{0} \cdot \varepsilon_{r, A} \cdot \varepsilon_{r, B}^{2}}{\left(d_{A} \cdot \varepsilon_{r, B}+d_{B} \cdot \varepsilon_{r, A}\right)^{2}} \cdot U_{0} \cdot u_{p}(t)$

Analogously, at the interface between material-B and electrode-2, the transient pressure $\widetilde{p}_{0, B-e_{2}}(t)$ is given by:

$\widetilde{p}_{0, B-e_{2}}(t)=-\frac{\mathcal{E}_{0} \cdot \varepsilon_{r, A}^{2} \cdot \varepsilon_{r, B}}{\left(d_{A} \cdot \varepsilon_{r, B}+d_{B} \cdot \varepsilon_{r, A}\right)^{2}} \cdot U_{0} \cdot u_{p}(t)$

At the interface between material-A and material-B, the electrostatic pressure is given by integrating the second term of (1):

$p_{0, A-B}=-\frac{\varepsilon_{0}}{2} \cdot \int_{\varepsilon_{r, A}}^{\varepsilon_{r, B}} E^{2} d \varepsilon_{r}$

Introducing the electric flux density $D$, where:

$D=\varepsilon_{0} \cdot \varepsilon_{r} \cdot E$

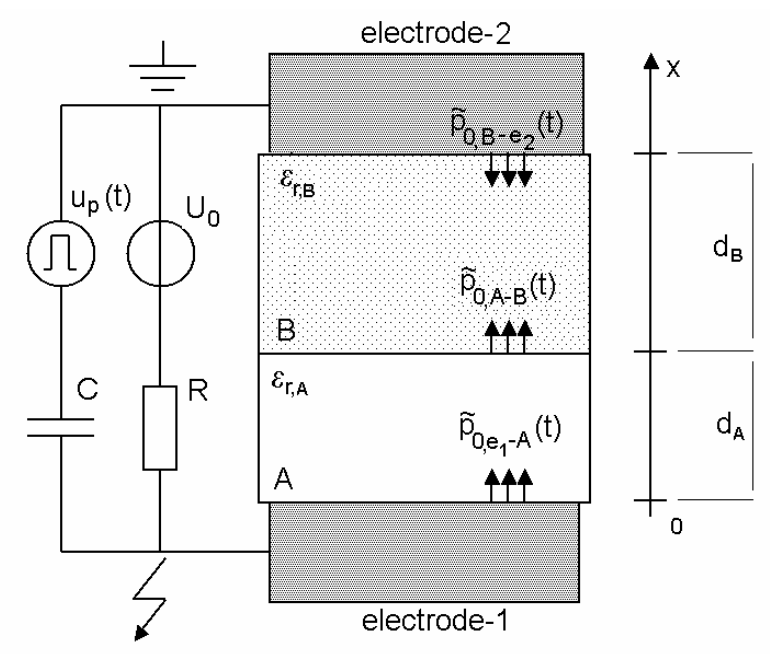

Figure 3. Electrically-induced pressure distribution in a multi-dielectric tested by means of the PEA method $\left(U_{0} \neq 0, \rho=0, \kappa=0\right)$. 
the pressure can be expressed as:

$p_{0, A-B}=-\frac{1}{2 \cdot \varepsilon_{0}} \cdot \int_{\varepsilon_{r, A}}^{\varepsilon_{r, B}} \frac{D^{2}}{\varepsilon_{r}^{2}} \cdot d \varepsilon_{r}=\frac{D^{2}}{2 \cdot \varepsilon_{0}} \cdot\left(\frac{1}{\varepsilon_{r, B}}-\frac{1}{\varepsilon_{r, A}}\right)$

Equation (12) can be rewritten in terms of time-dependent electric field:

$$
p_{0, A-B}(t)=\frac{1}{2} \cdot \varepsilon_{0} \cdot \varepsilon_{r, A}^{2} \cdot\left(\bar{E}_{0, A}+e_{p, A}(t)\right)^{2} \cdot\left(\frac{1}{\varepsilon_{r, B}}-\frac{1}{\varepsilon_{r, A}}\right)
$$

The transient component $\widetilde{p}_{0, A-B}(t)$ results:

$$
\widetilde{p}_{0, A-B}(t)=\frac{\varepsilon_{0} \cdot \varepsilon_{r, A}^{2} \cdot \varepsilon_{r, B}^{2}}{\left(d_{A} \cdot \varepsilon_{r, B}+d_{B} \cdot \varepsilon_{r, A}\right)^{2}} \cdot\left(\frac{1}{\varepsilon_{r, B}}-\frac{1}{\varepsilon_{r, A}}\right) \cdot U_{0} \cdot u_{p}(t)
$$

\subsection{SPACE CHARGE IN THE ABSENCE OF DC VOLTAGE AND INTERFACIAL CHARGE}

In Figure 4, the pressure distribution in the multi-dielectric is represented when space charge is present within material-A whereas interfacial charge and external dc voltage are absent.

The presence of a slab of low-mobility positive space charge of density $\rho_{A}$ and width $b_{A}$, where $b_{A}<<d_{A}$, which is embedded in the A-layer of the sample, affects the electrostatic pressure distribution within the sample. Firstly, a pressure wave is generated at the space charge location, $x_{\rho_{A}}$ in Figure 4. Its amplitude is given by:

$$
p_{\rho_{A}, x_{\rho A}}(t)=\rho_{A} \cdot b_{A} \cdot e_{p, A}(t)
$$

By inserting (2) in (15) the transient component $\widetilde{p}_{\rho_{A}, x_{\rho_{A}}}(t)$ of the pressure $p_{\rho_{A}, x_{\rho_{A}}}(t)$ becomes:

$$
\widetilde{p}_{\rho_{A}, x_{\rho_{A}}}(t)=\frac{\varepsilon_{r, B} \cdot \rho_{A} \cdot b_{A}}{\left(d_{A} \cdot \varepsilon_{r, B}+d_{B} \cdot \varepsilon_{r, A}\right)} \cdot u_{p}(t)
$$

A second consequence is that the multi-dielectric experiences an electric field $\bar{E}_{\rho_{A}}$ associated with the space charge $\rho_{A}$. By using (4) and (5), the field in the A-layer is given by:

$$
\begin{aligned}
& \bar{E}_{\rho_{A}, A}=-\frac{\rho_{A} \cdot b_{A} \cdot\left\lfloor\left(d_{A}-x_{\rho_{A}}\right) \cdot \varepsilon_{r, B}+d_{B} \cdot \varepsilon_{r, A}\right\rfloor}{\varepsilon_{0} \cdot \varepsilon_{r, A} \cdot\left(d_{A} \cdot \varepsilon_{r, B}+d_{B} \cdot \varepsilon_{r, A}\right)} \quad \text { if } 0<x<x_{\rho_{A}} \\
& \bar{E}_{\rho_{A}, A}=\frac{\rho_{A} \cdot b_{A} \cdot \varepsilon_{r, B} \cdot x_{\rho_{A}}}{\varepsilon_{0} \cdot \varepsilon_{r, A} \cdot\left(d_{A} \cdot \varepsilon_{r, B}+d_{B} \cdot \varepsilon_{r, A}\right)} \quad \text { if } x_{\rho_{A}}<x<d_{A}
\end{aligned}
$$

whereas in the B-layer the field is:

$$
\bar{E}_{\rho_{A}, B}=\frac{\rho_{A} \cdot b_{A} \cdot x_{\rho_{A}}}{\varepsilon_{0} \cdot\left(d_{A} \cdot \varepsilon_{r, B}+d_{B} \cdot \varepsilon_{r, A}\right)}
$$

When the pulsed field $e_{p}(\mathrm{t})$ is superimposed on the field $\bar{E}_{\rho_{A}}$, the pressure distribution is different from zero not only at the space charge location but also at the electrode/dielectric interfaces $\left(p_{\rho_{A}, e_{1}-A}(t)\right.$ and $\left.p_{\rho_{A}, B-e_{2}}(t)\right)$ and at the interface between the two different dielectrics $\left(p_{\rho_{A}, A-B}(t)\right)$ :

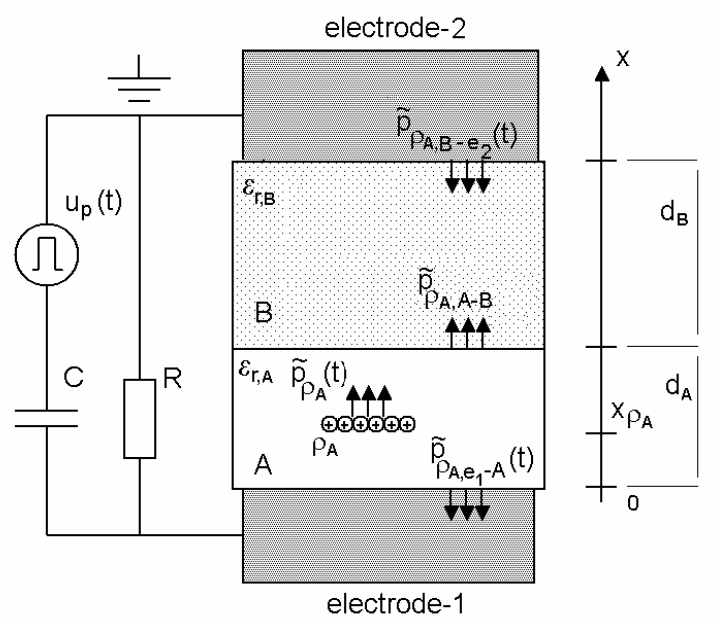

Figure 4. Electrically-induced pressure distribution in a multi-dielectric tested by means of the PEA method $\left(U_{0}=0, \rho \neq 0, \kappa=0\right)$.

$p_{\rho_{A}, e_{1}-A}(t)=\frac{1}{2} \cdot \varepsilon_{0} \cdot \varepsilon_{r, A} \cdot\left(\bar{E}_{\rho_{A}, A}+e_{p, A}(t)\right)^{2}$

$p_{\rho_{A}, B-e_{2}}(t)=-\frac{1}{2} \cdot \varepsilon_{0} \cdot \varepsilon_{r, B} \cdot\left(\bar{E}_{\rho_{A}, B}+e_{p, B}(t)\right)^{2}$

$p_{\rho_{A}, A-B}(t)=\frac{1}{2} \cdot \varepsilon_{0} \cdot \varepsilon_{r, A}^{2} \cdot\left(\bar{E}_{\rho_{A}, A}+e_{p, A}(t)\right)^{2} \cdot\left(\frac{1}{\varepsilon_{r, B}}-\frac{1}{\mathcal{E}_{r, A}}\right)$

The transient component of the pressures defined in (20-22) can be found by combining (2), (3), (17-22) and considering $E_{\rho}>>e_{p}$ :

$\widetilde{p}_{\rho_{A}, e_{1}-A}(t)=-\frac{\rho_{A} \cdot b_{A} \cdot \varepsilon_{r, B} \cdot\left\lfloor\left(d_{A}-x_{\rho_{A}}\right) \varepsilon_{r, B}+d_{B} \cdot \varepsilon_{r, A}\right\rfloor}{\left(d_{A} \cdot \varepsilon_{r, B}+d_{B} \cdot \varepsilon_{r, A}\right)^{2}} \cdot u_{p}(t)$

$\widetilde{p}_{\rho_{A}, B-e_{2}}(t)=-\frac{\rho_{A} \cdot b_{A} \cdot \varepsilon_{r, B} \cdot \varepsilon_{r, A} \cdot x_{\rho_{A}}}{\left(d_{A} \cdot \varepsilon_{r, B}+d_{B} \cdot \varepsilon_{r, A}\right)^{2}} \cdot u_{p}(t)$

$\widetilde{p}_{\rho_{A}, A-B}(t)=\frac{\rho_{A} \cdot b_{A} \cdot \varepsilon_{r, B} \cdot\left(\varepsilon_{r, A}-\varepsilon_{r, B}\right) \cdot x_{\rho_{A}}}{\left(d_{A} \cdot \varepsilon_{r, B}+d_{B} \cdot \varepsilon_{r, A}\right)^{2}} \cdot u_{p}(t)$

Similar expressions can be found in case of a slab of positive space charge $\rho_{B}$ of width $b_{B}$ embedded in the B-layer of the multi-dielectric at a distance $x_{\rho_{B}}$.

\subsection{PRESENCE OF INTERFACIAL CHARGE IN ABSENCE OF DC VOLTAGE AND SPACE CHARGE}

The pressure distribution in the multi-dielectric shown in Figure 5 is representative of the situation in which only interfacial charge is present in the multi-dielectric and no dc external voltage is applied.

If a positive surface charge $\kappa$ is present at the dielectric interface, a pressure wave $p_{\kappa, A-B}(t)$ is generated at the charge location:

$p_{\kappa, A-B}(t)=\frac{1}{2} \cdot \kappa \cdot\left[e_{p, A}(t)+e_{p, B}(t)\right]$ 
In (26), the surface charge $\kappa$ is assumed to experience the average pulsed field at the dielectric interface. Combining (2), (3) and (26), the transient component $\widetilde{p}_{\kappa, A-B}(t)$ of the pressure $p_{\kappa, A-B}(t)$ is obtained:

$\widetilde{p}_{\kappa, A-B}(t)=\frac{\varepsilon_{r, A}+\varepsilon_{r, B}}{2\left(d_{A} \cdot \varepsilon_{r, B}+d_{B} \cdot \varepsilon_{r, A}\right)} \cdot \kappa \cdot u_{p}(t)$

The electric field $\bar{E}_{\kappa}$ associated with the surface charge can be calculated by means of (4) and (5). In the A-layer the field is given by:

$$
\bar{E}_{\kappa, A}=-\frac{\kappa \cdot d_{B}}{\varepsilon_{0} \cdot\left(d_{A} \cdot \varepsilon_{r, B}+d_{B} \cdot \varepsilon_{r, A}\right)}
$$

whereas in the B-layer:

$$
\bar{E}_{\kappa, B}=\frac{\kappa \cdot d_{A}}{\varepsilon_{0} \cdot\left(d_{A} \cdot \varepsilon_{r, B}+d_{B} \cdot \varepsilon_{r, A}\right)}
$$

At the electrode/dielectric interfaces the pressures $p_{\kappa, e_{1}-A}(t)$ and $p_{\kappa, B-e_{2}}(t)$ are present:

$$
\begin{aligned}
& p_{\kappa, e_{1}-A}(t)=\frac{1}{2} \cdot \varepsilon_{0} \cdot \varepsilon_{r, A} \cdot\left(\bar{E}_{\kappa, A}+e_{p, A}(t)\right)^{2} \\
& p_{\kappa, B-e_{2}}(t)=-\frac{1}{2} \cdot \varepsilon_{0} \cdot \varepsilon_{r, B} \cdot\left(\bar{E}_{\kappa, B}+e_{p, B}(t)\right)^{2}
\end{aligned}
$$

By means of (2), (3), (28-31) and considering $E_{\kappa}>>e_{p}$, the transient components $\widetilde{p}_{\kappa, e_{1}-A}(t)$ and $\widetilde{p}_{\kappa, B-e_{2}}(t)$ become:

$$
\begin{aligned}
& \widetilde{p}_{\kappa, e_{1}-A}(t)=-\frac{\varepsilon_{r, A} \cdot \varepsilon_{r, B} \cdot \kappa \cdot d_{B}}{\left(d_{A} \cdot \varepsilon_{r, B}+d_{B} \cdot \varepsilon_{r, A}\right)^{2}} \cdot u_{p}(t) \\
& \widetilde{p}_{\kappa, B-e_{2}}(t)=-\frac{\varepsilon_{r, A} \cdot \varepsilon_{r, B} \cdot \kappa \cdot d_{A}}{\left(d_{A} \cdot \varepsilon_{r, B}+d_{B} \cdot \varepsilon_{r, A}\right)^{2}} \cdot u_{p}(t)
\end{aligned}
$$

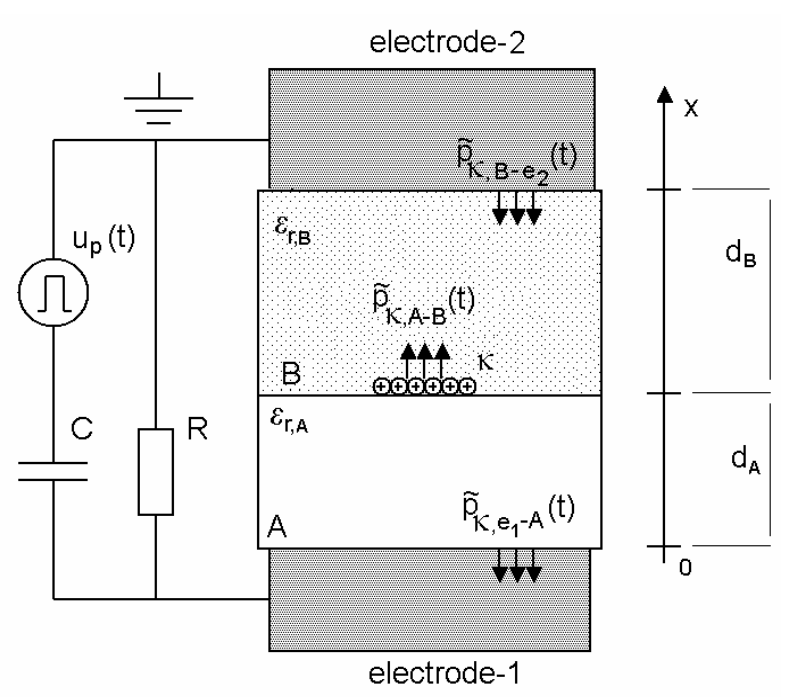

Figure 5. Electrically-induced pressure distribution in a multi-dielectric tested by means of the PEA method $\left(U_{0}=0, \rho=0, \kappa \neq 0\right)$.

\subsection{VOLTAGE APPLIED IN PRESENCE OF SPACE CHARGE AND INTERFACIAL CHARGE}

In general, the amplitude of the pressure wave distribution can be expressed as the superimposition of the pressure wave distributions obtained in the three particular situations studied before:

$\widetilde{p}(x, t)=\widetilde{p}_{0}(x, t)+\sum_{i} \widetilde{p}_{\rho_{i}}(x, t)+\widetilde{p}_{\kappa}(x, t)$

It is to be noted that for every considered case, the total pressure distribution, $p_{\text {TOT }}$, across the multi-dielectric is zero:

$p_{\text {TOT }}=\int_{0}^{d_{A}+d_{B}} f(x) \cdot d x=0$

\section{ACOUSTIC WAVE TRAVELING AND REFLECTION}

The acoustic waves calculated in the previous section are different from the detected waves at the sensor.

Firstly, acoustic waves traveling through an elastic medium are attenuated and dispersed. For situations where these effects are not negligible, procedures for recovering the original waveform from the attenuated/distorted signal have been developed [25]. Secondly, waves generated at different locations of the dielectric experience different generation, transmission and reflection coefficients. These phenomena will be studied for a multi-dielectric.

\subsection{ACOUSTIC WAVE PROPAGATION}

After their generation, pressure waves travel both in the direction of electrode- 1 and electrode- 2 . However, only those waves that travel towards electrode- 2 are detected by the sensor. Waves traveling toward electrode-1 do not reach the sensor, unless they are reflected at the interface between two different media. This will be discussed later.

The propagation of pressure waves originated during the testing of the multi-dielectric shown in Figure 6 is described in the following. The acoustic impedance of a material is defined as:

$Z_{i}=\delta_{i} \cdot v_{i}$

where $\delta_{i}$ is the mass density of the medium and $v_{i}$ the speed of sound in the medium. Considering the waves traveling through the multi-dielectric as planar waves, the generation coefficient $G$, the transmission coefficient $T$ and reflection coefficient $R$ can be calculated as [26]:

$$
\begin{aligned}
& G_{i-j}=\frac{Z_{j}}{Z_{i}+Z_{j}} \\
& T_{i-j}=\frac{2 \cdot Z_{j}}{Z_{i}+Z_{j}} \\
& R_{i-j}=\frac{Z_{j}-Z_{i}}{Z_{i}+Z_{j}}
\end{aligned}
$$

where the footer " $i$ " identifies the medium from which the wave comes from, while the footer " $j$ " identifies the medium toward which the wave is traveling. If an acoustic wave is 
originated at the interface between two media, " $i$ " and " $j$ ", the generation coefficient indicates the fraction of this wave traveling into medium " $j$ ". On the other hand, if an acoustic wave travels from medium " $i$ " towards medium " $j$ ", the transmission and reflection coefficients indicate the fractions of this wave which are respectively transmitted into medium " $j$ " and reflected back into medium " $i$ ".

In the situation depicted in Figure 6, the pressure wave $\widetilde{p}_{B-e_{2}}^{\prime}(t)$, which is the fraction of $\widetilde{p}_{B-e_{2}}(t)$ traveling into the sensor, can be calculated as follows:

$\widetilde{p}_{B-e_{2}}^{\prime}(t)=G_{B-e_{2}} \cdot T_{e_{2}-S} \cdot \widetilde{p}_{B-e_{2}}\left(t-\tau_{e_{2}}\right)$

At the sensor location, the wave arrives after a time $\tau_{e 2}$ from the firing of the pulse. $\tau_{e 2}$ is the time the acoustic waves take for traveling through electrode- 2 and is given by:

$\tau_{e_{2}}=d_{e_{2}} / v_{e_{2}}$

Expressions similar to (40) can be determined for $\widetilde{p}_{A-B}^{\prime}(t)$, and $\widetilde{p}_{e_{1}-A}^{\prime}(t)$ which are respectively the fraction of $\widetilde{p}_{A-B}(t)$

and $\widetilde{p}_{e_{1}-A}(t)$ traveling into the sensor:

$\widetilde{p}_{A-B}^{\prime}(t)=G_{A-B} \cdot T_{B-e_{2}} \cdot T_{e_{2}-S} \cdot \widetilde{p}_{A-B}\left(t-\tau_{B}-\tau_{e_{2}}\right)$

$\widetilde{p}_{e_{1}-A}^{\prime}(t)=G_{e_{1}-A} \cdot T_{A-B} \cdot T_{B-e_{2}} \cdot T_{e_{2}-S} \cdot \widetilde{p}_{e_{1}-A}\left(t-\tau_{A}-\tau_{B}-\tau_{e_{2}}\right)$

where:

$$
\begin{aligned}
& \tau_{A}=d_{A} / v_{A} \\
& \tau_{B}=d_{B} / v_{B}
\end{aligned}
$$

With regard to the acoustic waves due to the space charge $\rho_{A}$ within the bulk of the A-layer, the pressure wave $\widetilde{p}_{\rho_{A}}^{\prime}(t)$, traveling from the space charge location $x_{\rho_{4}}$ into the sensor is:

$\widetilde{p}_{\rho_{A}}^{\prime}(t)=\frac{1}{2} \cdot T_{A-B} \cdot T_{B-e_{2}} \cdot T_{e_{2}-S} \cdot \widetilde{p}_{\rho_{A}}\left(t-\tau_{\rho_{A}}-\tau_{B}-\tau_{e_{2}}\right)$

$\tau_{\rho_{A}}=\left(d_{A}-x_{\rho_{A}}\right) / v_{A}$

The coefficient $1 / 2$ in (46) is a direct consequence of (37).

The acoustic coefficients previously introduced are defined in case of two perfectly bonded media. However, materials adopted for PEA measurements present a finite surface roughness. So a non-ideal contact is present at the interface. The main consequence of this fact is that when an ultrasonic wave is normally incident to the interface, the acoustic coefficients will be frequency dependent [27]. In order to neglect this phenomenon, the surface roughness of the surfaces in contact must be much smaller then the wavelength of the acoustic waves traveling through the interface. In practice, to obtain this condition, surfaces in contact must be sufficiently smooth and/or a tiny amount of silicon oil can be used in order to improve the acoustic contact. However, if oil is used at the dielectric/dielectric interface, the multi-dielectric may exhibit a quite different space charge behavior from the situation in which the interface is dry [3-5].

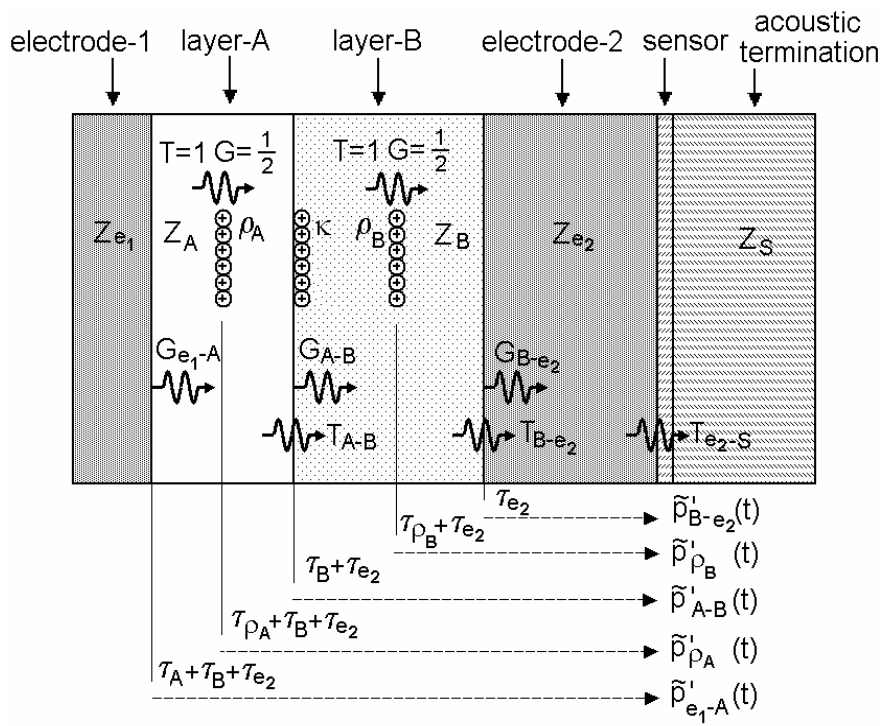

Figure 6. Propagation of acoustic waves in a multi-dielectric tested by means of the PEA method.

\subsection{ACOUSTIC WAVE REFLECTION}

In the case analyzed in Figure 7, the stress wave $\widetilde{p}_{B-e_{2}}^{\prime \prime}(t)$, which is the fraction of $\widetilde{p}_{B-e_{2}}(t)$ reaching the sensor after traveling through the B-layer and after being reflected back at the $\mathrm{A} / \mathrm{B}$ interface, is given by:

$\widetilde{p}_{B-e_{2}}^{\prime \prime}(t)=G_{e_{2}-B} \cdot R_{B-A} \cdot T_{B-e_{2}} \cdot T_{e_{2}-S} \cdot \widetilde{p}_{B-e_{2}}\left(t-2 \cdot \tau_{B}-\tau_{e_{2}}\right)$

Similarly, $\widetilde{p}_{\rho_{B}}^{\prime \prime}(t)$, which is the reflection at the A/B interface of the wave generated at the space charge $\rho_{B}$ location, becomes:

$\widetilde{p}_{\rho_{B}}^{\prime \prime}(t)=\frac{1}{2} \cdot R_{B-A} \cdot T_{B-e_{2}} \cdot T_{e_{2}-S} \cdot \widetilde{p}_{\rho_{B}}\left(t-2 \cdot \tau_{B}+\tau_{\rho_{B}}-\tau_{e_{2}}\right)$

$\tau_{\rho_{B}}=\left(d_{A}+d_{B}-x_{\rho_{B}}\right) / v_{B}$

However, these acoustic waves do not influence a measurement if they are detected by the sensor after the arrival of $\widetilde{p}_{e_{1}-A}^{\prime}(t)$ (wave generated at electrode- $1 /$ dielectric-A interface).

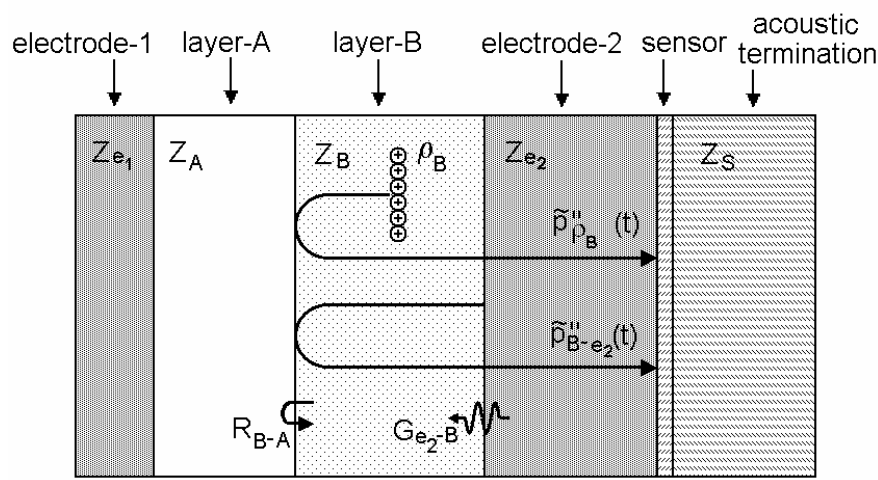

Figure 7. Reflection of acoustic waves in a multi-dielectric tested by means of the PEA method. 
In other words, the time it takes for a reflected wave to reach the sensor must be longer than the time it takes for the wave originated at the electrode-1/dielectric-A interface to arrive at the sensor. Then, no overlapping occurs if the following conditions are fulfilled:

$$
\begin{aligned}
& d_{B} / v_{B}>d_{A} / v_{A} \\
& \left(x_{\rho_{B}}-d_{A}\right) / v_{B}>d_{A} / v_{A}
\end{aligned}
$$

\section{EXPERIMENTAL 4.1 SAMPLES AND TEST PROCEDURES}

In this study, space charge measurements were performed on two different types of multi-dielectric. A multi-dielectric of the first type consisted of a sheet of polycarbonate (PC) in contact with a sheet of low density polyethylene (LDPE). A multi-dielectric of the second type was made of a plate of cross-linked polyethylene (XLPE) in contact with a plate of epoxy resin (Araldit ${ }^{\circledR}$ resin CW1483BD, filled with $47 \%$ Gwt Microdol). Neither lubricants nor treatment of any kind were applied at the dielectric interface. The particular materials chosen for this work, were selected because of the fact that their acoustic and electric properties are quite dissimilar, as shown in Table 1. In this way, the detected acoustic signal is expected to be quite different from the space charge distribution.

Measurements on PC-LDPE multi-dielectrics were performed by means of a PEA system equipped with a $9-\mu \mathrm{m}$ thick polyvinylidenefluoride (PVDF) sensor and a 60-dB gain amplifier with an input impedance of $50 \Omega$. The pulsed voltage was set to $300 \mathrm{~V}$, whereas the pulse width was $7 \mathrm{~ns}$. The multi-dielectric was placed in between a semicon HV electrode and an aluminum earth electrode. The LDPE side of the sample was connected to HV, whereas the PC side was connected to earth potential. A positive dc voltage of $2.75 \mathrm{kV}$ was applied to the multi-dielectric for a polarization time of $20 \mathrm{~s}$, during which voltage-on measurements were performed. After removing the dc poling voltage and short-circuiting the sample (these operations required a few seconds), voltage-off measurements were performed.

XLPE-epoxy multi-dielectrics were tested in a different PEA set-up, in which the PVDF sensor was $40-\mu \mathrm{m}$ thick, the amplifier gain was $40 \mathrm{~dB}$ and its input impedance $1.5 \mathrm{k} \Omega$. A 50 -ns wide pulse with amplitude of $2 \mathrm{kV}$ was used. A positive dc voltage of $23 \mathrm{kV}$ was applied to the multi-dielectric. Semicon electrodes were used. The XLPE side of the multidielectric was connected to $\mathrm{HV}$ and the epoxy side to earth. As in the previous case, the polarization time was $20 \mathrm{~s}$, during which voltage-on measurements were performed. The thickness of the semicon earth electrode was chosen in such a way that reflected acoustic waves at the interface between semicon earth electrode and aluminum PEA table did not overlap the waves representative of the pressure distribution inside the multi-dielectric.

All the measurements were done at room temperature. The short polarization time and the relatively low electric stress (the average electric field inside a tested multi-dielectric was $10 \mathrm{kV} / \mathrm{mm}$ ) guaranteed that neither space charge nor interfacial charge could build up during the voltage-on measurements.

Since the frequency response of the transducer amplifier system was not flat, deconvolution techniques $[9,28]$ were applied to the detected signal.

Table1. Some electric and acoustic properties of materials adopted for the PEA measurements.

\begin{tabular}{c|ccccc}
\hline material & $\begin{array}{c}\text { Speed of } \\
\text { sound } \\
{\left[\mathrm{m} \cdot \mathrm{s}^{-1}\right]}\end{array}$ & $\begin{array}{c}\text { Density } \\
{\left[\mathrm{kg} \cdot \mathrm{m}^{-3}\right]}\end{array}$ & $\begin{array}{c}\text { Acoustic } \\
\text { impedance } \\
{\left[\mathrm{kg} \cdot \mathrm{m}^{-2} \cdot \mathrm{s}^{-1}\right]}\end{array}$ & Thickness & $\begin{array}{c}\text { Relative } \\
\text { permittivity } \\
{[-]}\end{array}$ \\
\hline XLPE & 2000 & 900 & $1.8 \cdot 10^{6}$ & 1.5 & 2.3 \\
Epoxy & 2900 & 1600 & $4.6 \cdot 10^{6}$ & 0.8 & 4.5 \\
PC & 2300 & 1200 & $2.8 \cdot 10^{6}$ & 0.175 & 3.1 \\
LDPE & 2000 & 900 & $1.8 \cdot 10^{6}$ & 0.1 & 2.2 \\
Semicon & 2300 & 1100 & $2.5 \cdot 10^{6}$ & 1.2 & - \\
PVDF & 2260 & 1780 & $4.0 \cdot 10^{6}$ & $9 *-40^{* *} \cdot 10^{-3}$ & 8.5 \\
Al & 6420 & 2690 & $17.3 \cdot 10^{6}$ & $18^{*}-21^{* *}$ & - \\
\hline
\end{tabular}

* set-up for measurements on PC-LDPE multi-dielectrics

** set-up for measurements on XLPE-epoxy multi-dielectrics

\subsection{MEASUREMENT RESULTS}

In Figure 8, an example of a voltage-on signal detected on a space-charge free PC-LDPE multi-dielectric is presented. The signal clearly shows the presence of three peaks, which correspond to the electrostatic pressure at the electrodes and at the dielectric interface. It is to be noted that neither space charge nor interfacial charge were present within the multidielectric.

Figure 9 represents the voltage-off signal measured on a PCLDPE multi dielectric composed of a space charge-free LDPE sheet in contact with a PC sheet in which positive space charge is present. The PC sheet was in fact pre-stressed for 860 hours at $17.5 \mathrm{kV}$. After this electrical treatment, positive space charge was stably trapped within the PC.

In Figure 9, not only peaks at the electrodes and at the dielectric interface are visible, but also at the space charge location and within the LDPE-layer. It is to be noted that the LDPE is space charge free and neither external dc voltage is applied nor interfacial charge is present.

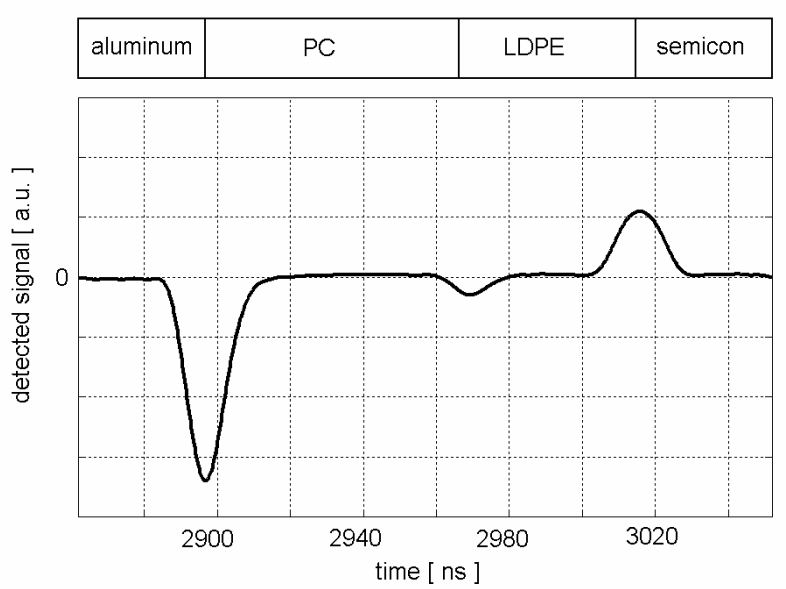

Figure 8. Acoustic signal in a space-charge free PC-LDPE multi-dielectric. Voltage-on measurement. 
In Figure 10, the voltage-on signal detected on a spacecharge free XLPE-epoxy multi-dielectric is shown. Several peaks are present in the signal. In addition to the peaks at both the electrodes and at the dielectric interface, two peaks within the XLPE-layer are notable (peak 1 and peak 2 in the figure). As in the case represented in Figure 8, neither space charge nor interfacial charge are present within the sample.

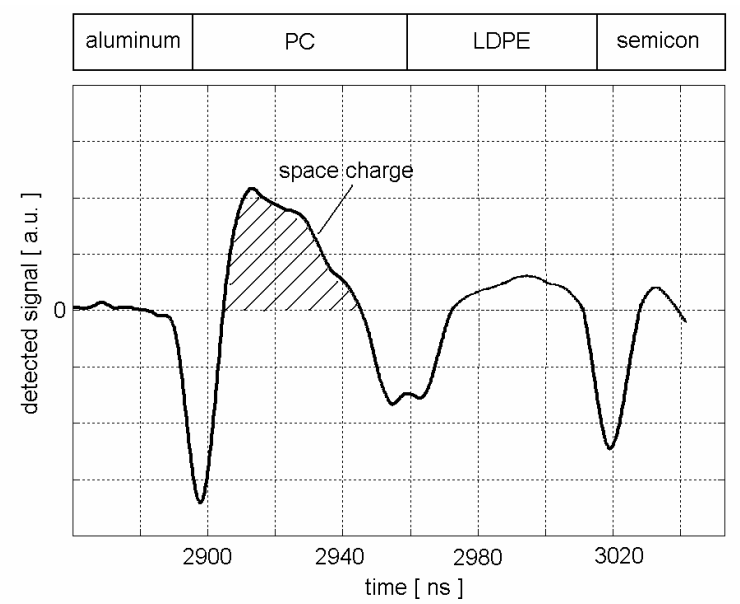

Figure 9. Acoustic signal in a PC-LDPE multi-dielectric, in which space charge is present within the PC. Voltage-off measurement; waveform not deconvolved.

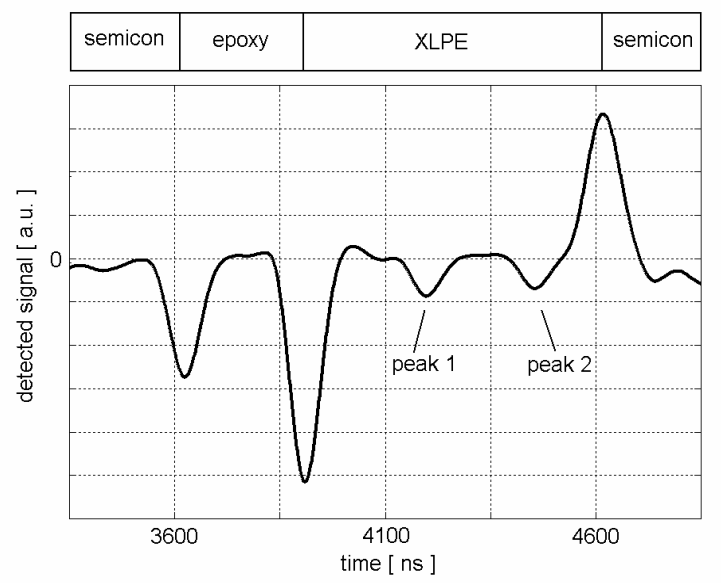

Figure 10. Acoustic signal in a space-charge free XLPE-epoxy multidielectric. Voltage-on measurement.

\section{DISCUSSION}

In order to interpret the acoustic signals represented in Figures 8, 9 and 10, the expected acoustic signal at the sensor was calculated. For this purpose, each layer of the multidielectric, the electrodes and the sensor were modeled as lossfree acoustic lines. Every acoustic line was characterized by its acoustic impedance and delay time, calculated as described in section 3.1. The electrically-induced pressure waves were modeled as pulsed pressure generators placed in the acoustic network as shown in Figure 11. The amplitude of the pulses was given according to (8), (9) and (14). In this way, the detected pressure signal in the space-charge free situation was derived.

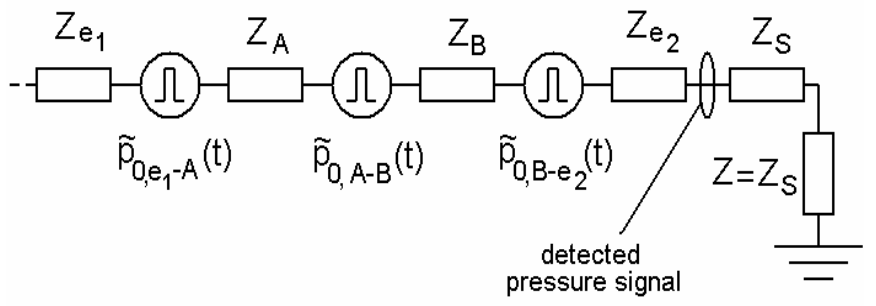

Figure 11. Acoustic network as used for the calculation of the pressure signal expected at the sensor. The acoustic impedance $\mathrm{Z}=\mathrm{Z}_{\mathrm{S}}$ relates to the acoustic termination.

Comparing the experimental patterns shown in Figures 8 and 10 to the result of calculations represented in Figures 12 and 13, the following can be said. The location and sign of the peaks in the measured signal correspond to those calculated. The magnitude of the measured peaks is also in good agreement with the calculation. Only the peak measured at the HV electrode showed amplitude smaller than that predicted by the calculation. The main reason for this is believed to be the attenuation and dispersion the acoustic waves generated at the HV electrode experience traveling through the material and through the dielectric interface.

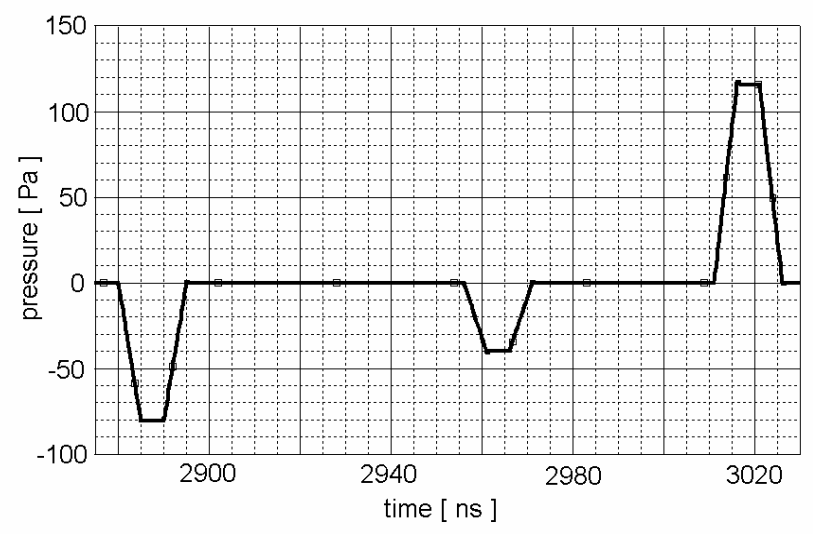

Figure 12. Expected acoustic signal at the sensor calculated for a PC-LDPE multi-dielectric. The multi-dielectric is assumed to be space-charge free.

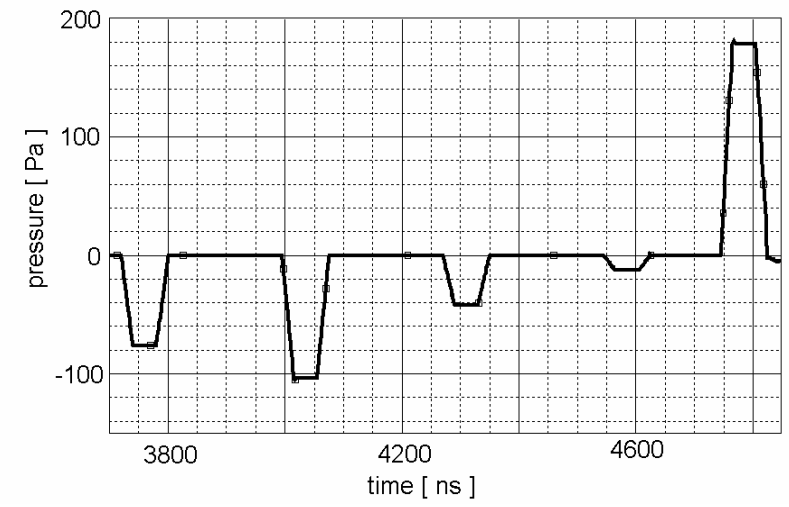

Figure 13. Expected acoustic signal at the sensor calculated for an XLPEepoxy multi-dielectric. The multi-dielectric is assumed to be space-charge free. 
The origin of the peak detected at the LDPE location in the signal shown in Figure 9 is to be attributed to reflection of acoustic waves at the PC-LDPE interface. So the signal within the LDPE does not represent any space charge. In fact, the pressure waves, which are generated at the space charge location and which travel toward the HV electrode, are reflected at the PC-LDPE interface and then detected at the sensor after traveling through the PC and the earth electrode.

The two peaks, which are detected between the dielectric interface and the HV electrode in the measured signal shown in Figure 10, are also due to reflection of acoustic waves and do not represent any space charge. Peak 1 is the reflection at the epoxy-XLPE interface of the fraction of the acoustic wave generated at the semicon-epoxy interface which travels toward the HV electrode. Peak 2 is the double reflection, first at the semicon-epoxy interface and then at the epoxy-XLPE interface, of the fraction of the acoustic wave generated at the epoxy-XLPE interface and traveling toward the earth electrode.

\section{CONCLUSIONS}

Generation, traveling and reflection of electrically-induced acoustic waves in a multi-dielectric tested by means of the PEA method were described in this paper. Based on a theoretical approach, procedures were developed for relating the space charge magnitude and location to the acoustic output signal given by a PEA system. Experimental investigations validated the proposed analytical methods.

Generally, when a multi-dielectric is tested, the detected acoustic signal does not correspond to the space charge distribution. There are two main reasons.

Firstly, the electrically-induced pressure distribution is different from the charge distribution if the permittivities of the layers composing the multi-dielectric are not the same. This leads to the presence of a pressure signal at the dielectric interface even in the absence of interfacial charge.

The second reason is that the detected acoustic signal does not correspond to the pressure distribution if the layers of the multi-dielectric have different acoustic properties. In fact, the magnitude of the acoustic waves is affected by the acoustic impedance of the materials. Besides, in case of acoustic mismatching, reflected waves may overlap the waves representing the pressure distribution inside the multidielectric.

Therefore, when the PEA method is applied to multidielectric test objects, particular attention should be paid to the interpretation of the detected signal. If the multi-dielectric is electrically and acoustically inhomogeneous, procedures should be used in order to collect a meaningful space charge pattern.

\section{REFERENCES}

[1] F.H. Kreuger, Industrial High DC Voltage, Delft University Press, 1995.

[2] M. Jeroense, M. Lindgren, A. Gustafsson and T. Schütte, "Interface charge in polymer laminates", Proc. IEEE Int. Conf. Cond. Breakdown Solid Dielectr., pp. 55-59, 1998.
[3] K.S. Suh, J.H. Nam, J.H. Kim, K.C. Ko and S.O. Han, "Interfacial properties of XLPE/EPDM laminates", IEEE Trans. Dielectr. Electr. Insul., Vol.7, pp. 216-221, 2000.

[4] R. Bodega, P.H.F. Morshuis, E. Redjosentono and J.J. Smit, "Dielectric interface characterization by means of space charge measurements", Proc. IEEE Conf. on Electr. Insul. Diel. Phenomena, pp. 728-733, 2003.

[5] K.S. Suh, J.Y. Kim, H.S. Noh and C.R. Lee, "Interfacial charge in polyethylene/ethylene vinylacetate laminates, IEEE Trans. Dielectr. Electr. Insul., Vol.3, pp. 758-764, 1996.

[6] M. Uchiumi, T. Tanaka, K. Ueda and M. Nitta, "Characteristics of space charge in polyethylene/ethylene-propylene-terpolymer laminates", Proc. Int. Symp. Electr. Insul. Mater., pp. 117-120, 1998.

[7] G. Chen, Y. Tanaka, T. Takada and L. Zhong, "Effect of polyethylene interface on space charge formation", IEEE Trans. Dielectr. Electr. Insul., Vol.11, pp. 113-121, 2004.

[8] T. Maeno, H. Kushibe, T. Takada and C.M. Cooke, "Pulsed electroacoustic Method for the measurement of volume charge in e-beam irradiated PMMA", Proc. IEEE Conf. Electr. Insul. Diel. Phenomena, pp. 389-397,1985.

[9] T. Maeno, T. Futami, H. Kushibe, T. Takada and C.M. Cooke, "Measurement of spatial charge distribution in thick dielectrics using the pulsed electroacoustic method", IEEE Trans. Electr. Insul., Vol. 23, pp. 433-439, 1988.

[10] T. Tanaka and M. Uchiumi, "Some consideration of voltage-induced interfacial space charge in several laminated dielectrics", Proc. IEEE Int. Conf. Cond. Breakdown Solid Dielectr., pp. 23-27, 1998.

[11] J. Beyer, P.H.F. Morshuis and J.J. Smit, "Investigation on charge and polarization effects at dielectric interfaces in laminated HVDC insulation", Proc. IEEE Conf. Electr. Insul. Diel. Phen., pp. 32-37, 1999.

[12] Y. Li and T. Takada, "Space charge distribution in multi-ply LDPE", Proc. IEEE Conf. Electr. Insul. Diel. Phenomena, pp. 397-402, 1992.

[13] T. Maeno and K. Fukunaga, "Space charge accumulation in multilayer insulators", Proc. IEEE Int. Symp. Electrets, pp. 207-210, 1996.

[14] H. Miyake, K. Iida, Y. Tanaka, T. Takada, T. Shindo and T. Tanaka, "Space charge formation at the interface between polyethylene and other polymeric materials", Proc. Conf. Propert. Applic. Dielectr. Materials, pp. 665-668, 2003.

[15] R. Bodega, P.H.F. Morshuis and J.J. Smit, "Space charge signal interpretation in dielectric combinations tested by means of the PEA method”, Proc. IEEE Int. Conf. Solid Dielectr., pp. 240-243, 2004.

[16] M. Wadamori, M. Fukuma, T. Maeno, K. Fukunaga and M. Nagao, "Proposal of numerical analysis model of acoustic wave propagation and generation on PEA method", Proc. Conf. Propert. Applic. Dielectr. Materials, pp. 863-866, 2003.

[17] M. Wadamori, M. Fukuma, M. Nagao, N. Hozumi and M. Kosaki, Y. Fukui, "Numerical analysis of acoustic wave generation and propagation on PEA method", Proc. Int. Symp. Electr. Insul. Mater., pp.403-406, 2001.

[18] J. Beyer, P.H.F. Morshuis and J.J. Smit, "Space charge phenomena in laminated HVDC insulation", Proc. Int. Symp. Electr. Insul. Materials, pp. 181-186, 1998.

[19] H. Miyake, Y. Tanaka, T. Takada and M. Hanai, "Characteristic of space charge distribution under DC stress in glass materials", Proc. IEEE Conf. Electr. Insul. Diel. Phenomena, pp. 128-131, 2000.

[20] J. Beyer, "Space Charge and Partial Discharge Phenomena in HVDC Devices", Ph.D.thesis Delft University of Technology, ISBN 90-6734097-9, 2002.

[21] W.K.H. Panofsky and M. Phillips, Classical Electricity and Magnetism, Chapter 6, Addison-Wesley, Reading, 1962.

[22] S. Holé, T. Ditchi and J. Lewiner, "Can non-destructive space charge measurement techniques have fallout in other fields?", Proc. IEEE Int. Symp. Electrets, pp. 32-35, 2002.

[23] S. Holé, T. Ditchi and J. Lewiner, "Non-destructive methods for space charge distribution measurements : what are the differences?", IEEE Trans. Dielectr. Electr. Insul., Vol. 10 No. 4, pp. 670-677, 2003.

[24] M.P. Cals, J.P. Marque and C. Alquié, "Application of the pressure wave propagation method to the study of interfacial effects in eirradiated polymer films", J. Appl. Phys, Vol. 72, No5, pp.1940-1951, 1992.

[25] Y. Li, K. Murata, Y. Tanaka, T. Takada and M. Aihara, "Space charge distribution measurement in lossy dielectric materials by pulsed electroacoustic method", Proc. Conf. Propert. Applic. Dielectr. Materials, pp. 725-728, 1994. 
[26] J. Blitz, Fundamentals of ultrasonics, Chapters 1 and 2, second edition, London butterworths, 1967.

[27] B. Drinkwater and P. Cawley, "Measurement of the frequency dependence of the ultrasonic reflection coefficient from thin interface layers and partially contacting interfaces", Ultrasonics 35, pp. 479-488, 1997.

[28] M. Jeroense, Charges and Discharges in HVDC Cables, Appendix C, PhD-thesis Delft University of Technology, Delft University Press, 1997.

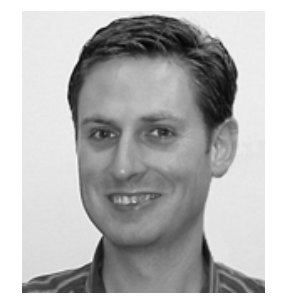

Riccardo Bodega was born in Lecco, Italy in 1976. $\mathrm{He}$ received the Master degree in electrical engineering from the Politecnico di Milano, Milan, Italy in 2002. In the same year, he joined the High Voltage Technology \& Management Institute at the Delft University of Technology, The Netherlands, where he started a Ph.D. program on HVDC polymeric-type cable systems. His main research interests concern the development of instruments for space charge measurements and the electrical behavior of dielectric interfaces.

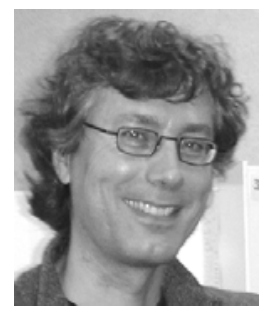

Peter H.F. Morshuis (M'95) was born in The Hague, The Netherlands, on 23 December 1959. In 1986, he received the Master degree in electrical engineering from Delft University of Technology. Between 1986 and 1988, he was involved in studies for NKF Kabel on the effects of defects on the lifetime of high voltage cables and in studies on new cable concepts. Since 1988, he has been a staff member of the High Voltage Group at Delft University of Technology where he was awarded the
Ph.D. degree in electrical engineering in 1993 on the topic of ultrawide-band electrical and optical studies of partial discharges in solid dielectrics. In 1998, he was a visiting Professor at the University of Bologna. Since 1999, he is an Associate Professor in High Voltage Engineering at Delft University of Technology and he is involved in teaching first year students in the field of Electricity and Magnetism and M.Sc. students in the field of High Voltage dc. His most important fields of interest are HVDC (materials and systems), space charge, partial discharge, and aging of electrical insulation. He is involved in a number of CIGRÉ activities and is an Associate Editor of the IEEE Transactions on Dielectrics and Electrical Insulation.

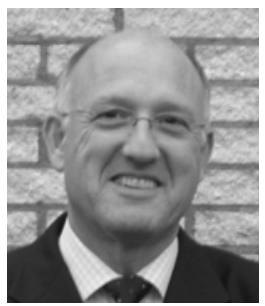

Prof. dr. Johan J. Smit (M’ 97), graduated in 1974 in experimental physics and in 1979 he received the Ph.D. degree at the State University of Leiden for his research in electro-magnetism on behalf of the National Science Foundation. He was employed in different research/management functions during two decades at KEMA's testing, consultancy and engineering company, Arnhem NL. Until 2003 he has been supervisory board member of the power grid company of South Holland. Since 1996 he is head of High Voltage Technology \& Management Institute at the Delft University of Technology. In 2004 he has become Director of Education in Electrical Engineering of the faculty of Electrical Engineering, Mathematics and Computer Science. In 2003 he has been the general chairmen of the XIII Conference ISH, "International Symposium on High Voltage Engineering" in Delft and he is currently chairing the ISH steering committee.

$\mathrm{He}$ is chairman of the executive board of the KSANDR, organisation for Knowledge Sharing and Research in the field of energy infrastructures. He represents the Netherlands in the Technical Committee of IEC 98 on Electrical Insulation Systems. He performs and has performed several functions in CIGRE, in particular the chairmanship of Study Committee D1, Materials and Emerging Technologies. In September 2004 he became honorary member of CIGRE, Paris. 